\title{
Effects of sign language experience on categorical perception of dynamic ASL pseudosigns
}

\author{
Catherine T. Best \\ University of Western Sydney, Penrith, New South Wales, Australia \\ and Haskins Laboratories, New Haven, Connecticut \\ Gaurav Mathur \\ Gallaudet University, Washington, D.C. \\ and Haskins Laboratories, New Haven, Connecticut \\ Karen A. Miranda \\ Massachusetts School of Professional Psychology, Boston, Massachusetts \\ AND \\ Diane LiLlo-Martin \\ University of Connecticut, Storrs, Connecticut \\ and Haskins Laboratories, New Haven, Connecticut
}

\begin{abstract}
We investigated effects of sign language experience on deaf and hearing participants' categorical perception of minimal manual contrast stimuli that met key criteria of speech perception research. A continuum of meaningless dynamic stimuli was created with a morphing approach, which manipulated videorecorded productions of phonotactically permissible pseudosigns differing between American Sign Language (ASL) handshapes that contrast on a single articulatory dimension ( $\mathrm{U}-\mathrm{V}$ : finger-spreading). AXB discrimination and AXB categorization and goodness ratings on the target items were completed by deaf early (native) signers (DE), deaf late (nonnative) signers (DL), hearing late (L2) signers (HL), and hearing nonsigners (HN). Categorization and goodness functions were less categorical and had different boundaries for DL participants than for DE and HL participants. Shape and level of discrimination functions also differed by ASL experience and hearing status, with DL signers showing better performance than DE, HL, and especially HN participants, particularly at the U end of the continuum. Although no group displayed a peak in discrimination at the category boundary, thus failing to support classic categorical perception, discrimination was consistent with categorization in other ways that differed among the groups. Thus, perception of phonetic variations underlying this minimal sign contrast is systematically affected by language experience.
\end{abstract}

Sign languages are naturally occurring languages used by members of deaf communities throughout the world. Although they capitalize on visible actions of the hands and arms instead of audible actions of vocal tract articulators, established sign languages display the full array of hierarchically organized linguistic levels found in spoken languages (Sandler \& Lillo-Martin, 2006). The interest of the present research was in sign-speech correspondence in the perceptual effects of language experience at the foundational level of linguistic structure - specifically, the phonological level: formational elements that provide coherent, systematic internal structure to a language's basic units of meaning (words or morphemes). The internal structure of words in spoken languages appears as rule-governed combinations of consonants and vowels (phonological segments). Signs have analogous internal phonological structure, instantiated as systematic combinations of manual formational properties (Brentari, 1998; Liddell \& Johnson, 1989; Sandler, 1989). Each sign language employs its own rule-governed combinations of a constrained subset of contrastive manual features as the basis for linguistically permissible forms in its lexical inventory. These formational features are described according to three or four parameters: handshape, location, movement, and, in some approaches, orientation (Battison, 1978; Stokoe, Casterline, \& Cronberg, 1965). Handshape refers to specific configurations of fingers and thumb; for example, in the V handshape of American Sign Language (ASL), the index and middle fingers are extended and spread, but the thumb and other fingers are closed (completely flexed). Location indicates a position on the face, body, or area in front of the signer to or from which the hand moves-for example, when the dominant hand contacts the nondominant hand's palm. Movement describes the action that the hand/arm 
performs - for example, a single arced movement between two locations. Orientation specifies the direction the palm is facing, relative to the signer or in a 3-D Cartesian system in front of the signer, as when, for example, the dominant hand's palm faces the signer.

These manual formational parameters are thought to function in signed languages in a fashion analogous to the phonetic feature classes used in linguistic analysis of phonological segments in spoken languages. In both language modalities, the basic formational, or phonetic, features serve as the organizational pivot for minimal phonological contrasts; for example, English /f/ and /v/ differ only in whether, during consonant production, the vocal cords are held open to prevent voicing or allowed to vibrate and thus produce voicing. ASL handshapes $\mathrm{V}$ versus $\mathrm{U}$ differ only in whether the extended index and middle fingers are spread apart or held together. Minimal phonological contrast permits words and signs to be meaningfully distinguished by a single critical feature. For example, English pat versus bat differ only in the voicing feature of their initial stop consonants, whereas ASL signs CANDY and APPLE differ minimally in handshape - that is, whether the index finger of the otherwise closed dominant hand is extended (1-index handshape) or flexed (X handshape; see Figure 1).

The obvious differences between manual-visual and vocal-auditory transmission modalities, however, raise fundamental questions about perception of the phonetic/ formational features in signs, as compared with speech. To what extent does perception of signs and speech diverge as a result of constraints related to their different primary perceptual modalities? Conversely, how might the apprehension of minimal contrast in the two language classes reflect the essentially amodal linguistic organization that provides hierarchical structure for both language modalities? And, following from the latter question, how might language experience modulate perception of sign, as compared with speech, contrasts?

We approached these questions from the vantage point of three striking and related characteristics often reported and widely accepted for speech perception: (1) Minimal phonological contrasts tend to be perceived categorically, but (2) this tendency varies widely across phonetic classes, and (3) several key parameters of categorical perception are modulated by specific language experience. Therefore, the present research assessed categorical perception of a minimal sign contrast and examined how it is influenced by varying experience with the target sign language, ASL, and/or with spoken English.

In categorical perception, perceivers display a sharp boundary in differential categorizations of tokens along a stimulus continuum that varies in equal physical steps between minimally contrasting endpoints. Correspondingly, they also display a correlated peak in discrimination for token pairs that straddle the boundary, with significantly poorer discrimination of equidistant within-category token pairs. Although there has been extensive debate about whether or not this categorical pattern provides unequivocal evidence of a human specialization for language (e.g., Liberman, Cooper, Shankweiler, \& Studdert-Kennedy, 1967, vs. Diehl \& Kluender, 1987; for broad coverage, see Harnad, 1987), this metatheoretical issue was not our primary concern. Rather, we employed categorical perception as a sensitive tool for assessing differences in perception of phonetic variations between minimally contrasting sign targets, among viewers who vary in specific language experience (see also Hallé, Best, \& Levitt, 1999). In other words, we were not seeking to answer the fundamental binary question of whether sign contrasts are categorically perceived; rather, our focus was on relative differences in the categoricity of the perception of sign contrasts (steepness and location of category boundaries, goodness judgments of tokens throughout the continuum, shape of discrimination functions, within-category discrimination) by several perceiver groups that differed systematically in their language experience. Prior research on categorical perception indicates that, indeed, degree of categoricity in speech perception does vary both across phonetic contrasts (intrinsic properties) and across perceiver groups with differing language experience (extrinsic factors).

With respect to types of phonetic contrasts, highly categorical labeling and discrimination have been found for the perception of stop consonant voicing and place of articulation contrasts (e.g., Joanisse, Zevin, \& McCandliss, 2007; Liberman et al., 1967; Liberman, Harris, Hoffman, \& Griffith, 1957; McQueen, 1996; Simos et al., 1998). However, categoricity is somewhat weakened for fricatives (shallower boundaries and discrimination peaks), and weaker still for approximants such as $/ \mathrm{r} /-/ \mathbf{l} /$ (e.g., Ferrero, Pelamatti, \& Vagges, 1982; Rosen \& Howell, 1987). Perception of steady-state vowel contrasts is still less categorical, or even
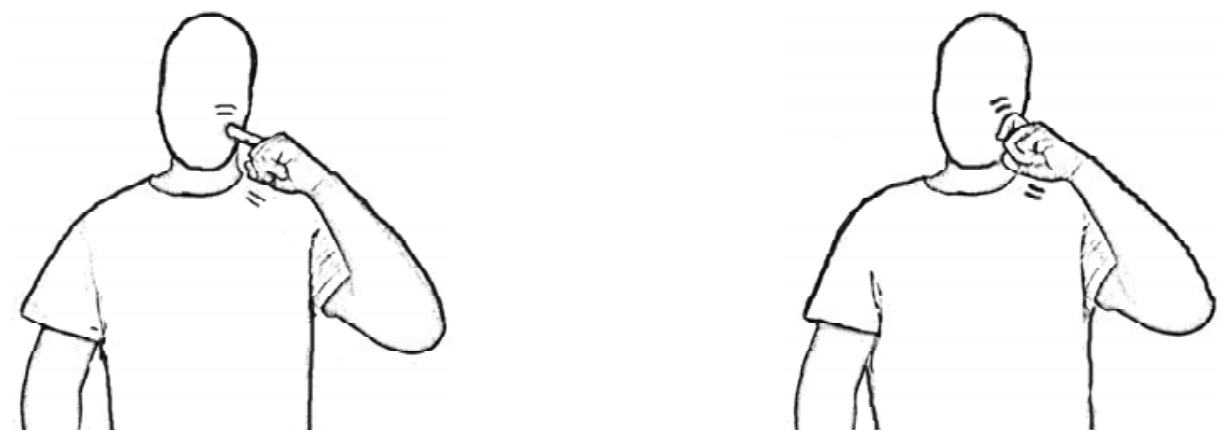

Figure 1. CANDY versus APPLE minimal-handshape contrast in ASL. 
A



1

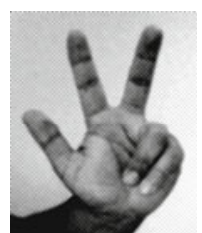

3



Open-N

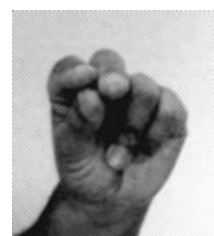

Closed-N

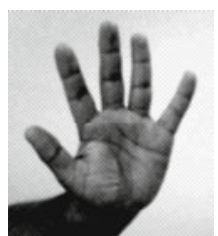

5

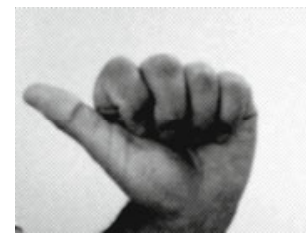

A-Bar

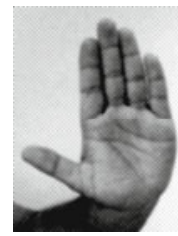

B-Bar

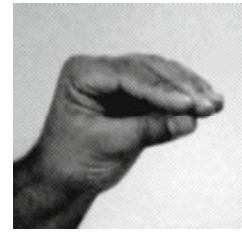

Flat-O

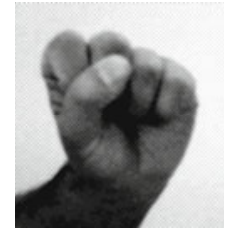

$\mathrm{S}$

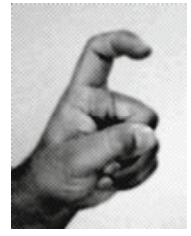

X

B


Figure 2. (A) Handshapes: 1, 3, open-N, closed-N, 5, A-bar, B-bar, flat-O, S, X. (B) Locations: Upper cheek, back jaw, chin, and neck (produced with 1-index handshape).

continuous. Vowel category boundaries are shallow, withincategory discrimination is high, and the discrimination peak at the category boundary is attenuated or lacking, unless unnaturally brief vowels are used (Fry, Abramson, Eimas, \& Liberman, 1962; Fujisaki \& Kawashima, 1969; Pisoni, 1973; Repp, Healy, \& Crowder, 1979; Stevens, Liberman, Öhman, \& Studdert-Kennedy, 1969).

Clearly, then, categorical perception is not equivalent across all speech contrasts, instead becoming progressively softened as the stimulus information for the contrasting segments increases in intensity and/or extension over time. This is potentially relevant to categorical perception of sign contrasts, given that "single-syllable" monomorphemic signs are generally longer in duration than monosyllabic spoken words are. Moreover, sign formational parameters tend to be coproduced, all of them extending throughout a sign rather than appearing as a sequence of shorter, overlapping subunits, as in spoken words. Thus, categorical perception of sign contrasts might be attenuated relative to stop consonant perception - that is, more like vowels.

Moreover, the pattern and degree of categorical perception for speech contrasts is modulated not only by intrinsic phonetic variations among phonological elements, but also by the extrinsic factor of variations in language experience. Location, steepness, and/or presence of category boundaries, as well as height/presence of peaks and level of within-category performance in discrimination, deviate markedly among listener groups whose native languages differ in either the phonological presence/absence or the phonetic realization of the target contrasts tested. Languagespecific group differences in categorical perception have been found for stop consonant voicing distinctions (voice onset time; e.g., Abramson \& Lisker, 1970; Keating, Mikoś, \& Ganong, 1981; Simon \& Fourcin, 1978; Williams, 1977), place of articulation distinctions (Werker \& Lalonde, 1988), and approximant place distinctions (Best \& Strange, 1992; Hallé et al., 1999; MacKain, Best, \& Strange, 1981; Miyawaki et al., 1975). Importantly, language-specific perceptual effects have also been found for phonological distinctions that are perceived much less categorically: vowels (Stevens et al., 1969) and lexical tone contrasts. For example, native Mandarin speakers perceive Mandarin lexical tone contrasts more categorically than do French speakers, even though the pattern of categorization and labeling for Mandarin listeners themselves is not strongly categorical, being more akin to vowel than to stop perception (Hallé, Chang, \& Best, 2004; see also Chang, Hallé, Best, \& Abramson, 2008; Francis, Ciocca, \& Kei, 2003). Thus, the categorical perception paradigm provides sensitive measures of the impact of variations in language experience, despite intrinsic phonological class variations in categoricity.

There are only four existing published reports on categorical perception of manual contrasts in sign. In the first (Newport, 1982), handshape (1 vs. X; see Figure 2) and location (chin vs. upper cheek [corner of eye], see Figure 2) 

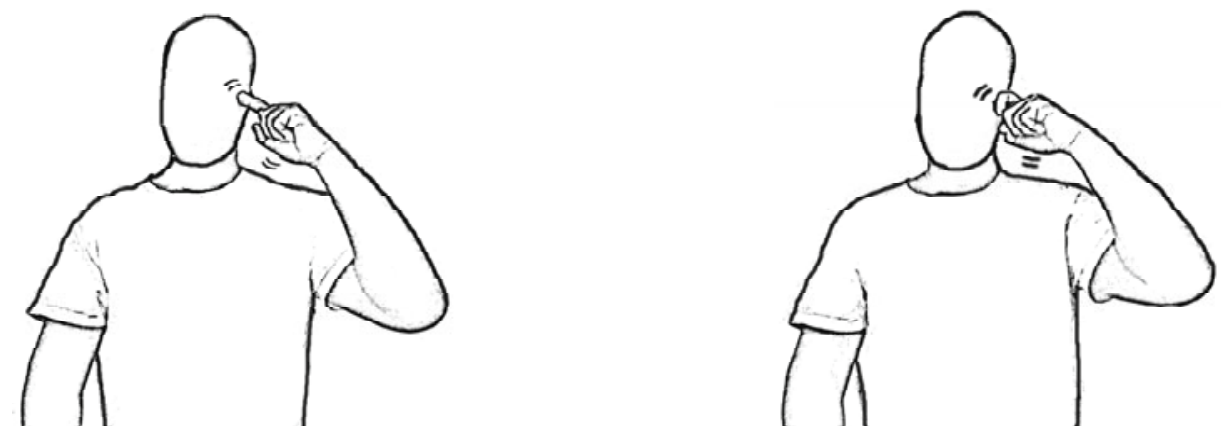

Figure 3. CHINESE versus ONION minimal-handshape contrast in ASL.

contrasts among the ASL signs CANDY, APPLE (Figure 1), CHINESE, and ONION (Figure 3) were examined in categorical perception experiments that used carefully selected video recordings of a deaf native signer producing incremental variations in handshape and location between the endpoint signs; the researchers also used static line drawings based on still frames from the videos. Deaf native ASL signers showed relatively categorical labeling functions for both the dynamic videos and the static line drawings, but their discrimination lacked clear boundary peaks and was quite good within categories, similar to findings with naturallength vowels.

In a recent report, Morford, Grieve-Smith, MacFarlane, Staley, and Waters (2008) tested perception of handshape (A-bar vs. B-bar; B-bar vs. claw; and flat-O vs. 8) and location (chin vs. upper cheek and chin vs. chest) contrasts using dynamic, synthetic stimuli with meaningful ASL signs as the endpoints (see Figures 2A and 2B for some of these contrasts). They compared three groups with respect to identification and discrimination of these stimuli: deaf native ASL signers, deaf nonnative ASL signers who had acquired ASL after the age of 10 , and hearing nonnative signers who similarly had learned ASL after the age of 10. Although Morford et al. did not find any difference in identification performance, they found effects of language experience on the discrimination of the handshape stimuli. Both deaf late signers and hearing late signers showed better discrimination than did the deaf native signers at the category boundaries. However, the deaf native signers showed the largest difference between within- and across-category judgments, because they had poorer within-category discrimination than the other two groups did.

The other two articles claimed that there is categorical perception, but only for some and not other ASL contrasts, and only in native signers. The third report (Emmorey, McCullough, \& Brentari, 2003) used static handshape and location contrasts between actual ASL signs, generated with computer animation software, which allowed creation of precise physically equal continuum steps. For each parameter, they tested two contrastive distinctions and one noncontrastive manual one: handshape (see Figure 2A; contrastive, A- vs. B-bar and 5 vs. 3; noncontrastive or allophonic, closed- vs. open-N), and location (see Figure 2B; contrastive, chin vs. upper cheek as in Newport [1982], and back jaw (ear) vs. neck; noncontrastive/allophonic, back jaw vs. chin). The authors concluded that deaf native ASL signers showed categorical perception for both contrastive handshape distinctions, but hearing sign-naive participants did not. By comparison, neither group showed categorical perception for either location contrast or for either type of allophonic distinction.

The fourth study (Baker, Idsardi, Golinkoff, \& Petitto, 2005) differed importantly from the other three in its use of nonsense stimuli rather than real signs; it also differed from Emmorey et al. (2003) and the follow-up experiments of Newport (1982) by using dynamic video stimuli. Baker et al. employed three handshape contrasts (A- vs. B-bar, as in Emmorey et al., 2003, as well as 5 vs. flat-O and 5 vs. S; see Figure 2A). A native ASL signer articulated a carefully controlled and verified series of handshape variants between the endpoints of each contrast, based on precise measurements of finger-palm distances. She produced each variant with her dominant (right) hand held upright about level with her ipsilateral shoulder, and slowly $(1.5 \mathrm{~Hz})$ rotated her palm orientation from facing the camera to facing the signer. The endpoint tokens were meaningless in ASL. Participants completed a categorization task in which the endpoint video tokens were repeated for $6 \mathrm{sec}$ (i.e., $\sim 8$ rotations of palm orientation) at the beginning of each test block, as well as a same-different (AX) discrimination task in which each token was presented for $1.5 \mathrm{sec}(\sim 2$ hand rotations). Deaf native signers showed categorical perception for the A- versus B-bar contrast, consistent with Emmorey et al., and for the 5 versus flat-O contrast, but not for the 5 versus $\mathrm{S}$ contrast. Hearing sign-naive participants failed to exhibit categorical perception for any contrasts.

Thus, for deaf native ASL signers, categorical perception has been claimed for some handshape contrasts (A- vs. B-bar: static, Emmorey et al., 2003; dynamic, Baker et al., 2005) by some researchers but not by others (Morford et al., 2008), whereas, conversely, a lack of categorical perception has been claimed for other handshape contrasts ( 1 vs. X contrast: dynamic and static, Newport, 1982; 5 vs. S contrast: dynamic, Baker et al., 2005; and 5 vs. 3 contrast as well as closed-N vs. open-N allophones: static, Emmorey et al., 2003; B-bar vs. claw, and flat-O vs. 8: dynamic, Morford et al., 2008). None of the location contrasts tested thus far has shown categorical perception in signers (static, Emmorey et al., 2003; dynamic and static, Newport, 1982; dynamic, Morford et al., 2008). 
Sign-naive participants allegedly lack categorical perception for all sign contrasts tested (dynamic, Baker et al., 2005; static, Emmorey et al., 2003).

Gaps thus remain in our understanding of categorical perception of sign contrasts, with respect to both intrinsic (sign properties) and extrinsic (language experience) contributions. Indeed, there is much we still don't know, even about native signers' perception. Direct comparison of the four sets of results is difficult, because the reports used different stimulus and design approaches; only Newport (1982) used predictions of discrimination functions from categorization. Inspection of published discrimination functions also suggests inconsistent interpretations of categoricity. Specifically, the general shape and level of discrimination functions are fairly similar (and shallow rather than steeply peaked) on A-bar versus B-bar for hearing controls, said to lack categorical perception (-) (Emmorey et al., 2003), and for native signers, who were said to display categorical perception $(+)$ of that contrast (Baker et al., 2005; Emmorey et al., 2003); but [-] in Morford et al., 2008), as well as on 5 versus flat-O (Baker et al., 2005). Conversely, signers' discrimination function on A-bar versus B-bar $(+)$ differs from that for 5 versus 3 , which yielded rather similar discrimination functions in signers $(+)$ and controls $(-)$, despite opposing claims about categoricity (Emmorey et al., 2003). Finally, both signers' (-) and controls' $(-)$ discrimination functions on upper cheek versus chin (Emmorey et al., 2003) appear similar to those of signers (Emmorey et al., 2003) on 5 versus 3 (+) and those of signers (Baker et al., 2005) on 5 versus S (-), as well as on all continua (-) in Newport and Morford et al. Only the discrimination patterns for signers $(+)$ on A- versus B-bar (Baker et al., 2005; Emmorey et al., 2003) and for signers (+) on 5 versus flat-O (Baker et al., 2005) would be considered categorical in shape by analogy to speech perception findings, but then this would have to be said for nonsigners (-) on A- versus B-bar (Baker et al., 2005; Emmorey et al., 2003). Moreover, even when performance reflected some degree of categorical perception, the relative shallowness of the category boundaries and good within-category discrimination were more in line with the weakly categorical perception seen for vowels than with the clearly categorical patterns found for stop consonants.

Attributes of the stimuli (intrinsic) may have contributed to weak, inconsistent categoricity. In two studies, one or all stimulus continua were static displays of some handshape at some location (Emmorey et al., 2003; Newport, 1982). Static two-parameter manual configurations are not phonotactically permissible as signs, which require movement as well as handshape and location features. Such displays are, therefore, not ideal as analogs to the phonotactically permissible nonsense syllables used in speech studies. Two sign studies, however, used one or more dynamic stimulus series composed of naturally produced manual forms selected from among variants produced by the signers as incremental changes between the contrastive endpoints (Baker et al., 2005; Newport, 1982). Although the items were carefully chosen on the basis of measurements of the signer's fingers and palm, none of the final continua were computer controlled, leaving open two possible sources of variation that might have affected stepto-step performance differences: residual token-to-token variations on noncriterial (unmeasured) dimensions and/ or fine-grained residual imprecision in step sizes along the criterial dimension(s). Baker et al.'s dynamic nonsense stimuli also deviate from ASL phonotactic requirements in two ways: Permissible sign forms must have only one or two repetitions (not six), and if they involve a change in palm orientation, it should be fairly rapid (not slowly rotating). On the other hand, whereas real signs (Newport, 1982) are, obviously, phonotactically permissible, they introduce the potential confound of lexical frequency biases on categorical perception (see Ganong, 1980). Given their aim to identify perception of phonetic contrasts per se, most categorical speech perception studies have used permissible nonword contrasts in order to minimize lexical biases, and have used computer-controlled stimulus generation to ensure physically equal step sizes and to eliminate other extraneous variation along the continuum. Morford et al. (2008) did use a computer-synthesized dynamic sign continuum; however, the signs were meaningful, leaving open the possibility of lexical bias, as they acknowledged.

Using lexical items would also confound interpretation of variations in sign perception among groups differing in language experience, a core interest of the present study, given that signers would know the meaning of lexical signs, whereas sign-naive perceivers would not. Two of the previous studies evaluated language experience effects by comparing deaf native ASL signers with hearing signnaive perceivers on categorical perception of nonlexical sign contrasts (Baker et al., 2005; Emmorey et al., 2003). The groups did differ in terms of performance; however, it is difficult to discern the responsible factor, because they differed not only in ASL experience, but also in native language (first language [L1]) modality, hearing status, and, relatedly, in speech experience. We therefore added to the present study a hearing group who were nonnative second language (L2) learners of ASL, for comparison with a sign-naive hearing group, in order to evaluate the impact of sign language experience while keeping hearing status and speech experience comparable. The sign-fluent hearing group were late (postadolescent) learners of ASL, whom we also compared with a new fourth group unique to the deaf community: deaf late nonnative learners of sign language. Only a small minority of signers acquire ASL from birth from deaf, ASL-using parents. Over $90 \%$, born to hearing families, learn ASL as a nonnative language from deaf peers later, usually after first being exposed to English visual-oral/reading training, which often fails to yield optimal English proficiency due to impaired auditory access to the language. Comparison of hearing versus deaf nonnative signers allowed us to probe the impact of acquiring ASL after establishing a highly proficient versus a more limited L1 foundation in spoken English (see Mayberry, 1993). Also, comparison of native versus nonnative lateonset deaf signers allowed assessment of ASL onset-age effects with hearing status held constant. ${ }^{1}$

Given that both speech and signs have phonological structure, one hypothesis is that native phonological structure will affect perception of contrasts similarly by users 
of the two types of language. Categorical perception is one measure of how phonological structure affects perception of contrasts. However, as mentioned above, there is variation in categorical perception of speech, associated with its two major phonological classes, consonants and vowels. Stop consonants are perceived most categorically in the classic sense, but monophthongal vowels are perceived much less categorically. If sign contrasts function in perception for signers as speech contrasts do for spokenlanguage users, we might expect to see perceptual patterns like those seen in speech perception, but we do not yet know whether sign contrasts would be perceived more like stop consonant contrasts or like vowel contrasts, or somewhere in between, as with approximant contrasts in speech. Alternatively, sign contrasts may differ qualitatively from speech contrasts at the perceptual level, because of various intrinsic stimulus factors, including the modality difference itself. In this case, we would expect some aspect of performance on a categorical perception task to differ from anything seen with any class of speech contrasts.

With respect to potential language experience effects, developmental speech findings have shown that native phonological structure is learned very early by hearing infants. If phonological structure does affect perception of sign, an experiential hypothesis would be that early acquisition should likewise result in optimal perceptual tuning to that structure; that is, deaf early (native) signers should show the clearest categorical labeling of a sign contrast and good discrimination at the category boundary but poor discrimination within each category, whereas deaf late (nonnative) signers should show poorer categorization and/or a different pattern or mean level of discrimination. Both hearing late (nonnative) signers and hearing nonsigners should also show perceptual differences from native deaf signers, since they also lack early exposure to ASL. However, consistent with findings that native language experience dramatically affects listeners' perception of nonnative speech contrasts (e.g., Best, McRoberts, \& Goodell, 2001; Hallé et al., 1999; Miyawaki et al., 1975), the hearing groups could show some type of impact of their native English experience on perception of sign contrasts, possibly showing (somewhat) categorical perceptual patterns. Thus, both hearing groups' performance could differ from that of the deaf nonnative group, whose English is less strongly established. However, evidence of improved L2 speech perception in more experienced late L2 learners (e.g., MacKain et al., 1981) additionally suggests that the hearing nonnative signers are likely to differ in some way from the hearing naive group, due to their L2 experience with ASL.

The prior categorical sign perception studies followed categorical speech perception research methods to some extent, but none met all of the potentially relevant stimulus and task characteristics of the speech studies. Therefore, to evaluate the hypotheses discussed above regarding perceptual and experiential influences, we took a new approach to stimulus generation, developing stimuli that were (1) dynamic, (2) phonotactically permissible (3) nonsense pseudosigns, (4) created under computer control to ensure precise equality of step sizes (5) along a continuum distinguished by a minimal ASL handshape distinction, which (6) reflected a single contrastive articulatory difference and (7) displayed greater visual similarity than most of the previous studies' sign contrasts did. We employed AXB procedures for our categorization and discrimination tasks to level the playing field by reducing memory load (see, e.g., Best et al., 2001; MacKain et al., 1981), particularly for naive perceivers who lack labels for the endpoints of an unfamiliar contrast (see, e.g., Bailey \& Summerfield, 1977; Best, Morrongiello, \& Robson, 1981; Best, Studdert-Kennedy, Manuel, \& RubinSpitz, 1989). To maximize detection of group differences in sensitivity to gradient within-category phonetic variation, we also had participants make goodness-of-fit ratings following their categorization judgments (see Hallé et al., 1999; Hodgson \& Miller, 1996; Iverson \& Kuhl, 1996; Kuhl, Williams, Lacerda, Stevens, \& Lindblom, 1992; Miller, 1994).

\section{METHOD}

\section{Participants}

There were 10 participants per group: deaf early signers (DE), deaf late signers (DL), hearing late signers (HL), and hearing signnaive perceivers $(\mathrm{HN})$. Each group's gender distribution, mean and range of age at the time of the experiment, mean and range of onset age of acquisition (AoA) of ASL, and mean and range of length of experience with ASL are given in Table 1.

All deaf participants were profoundly deaf in both ears from 2 years of age or earlier. DE signers all had deaf parents and were exposed to ASL from birth, whereas DL signers all had hearing parents and started learning ASL after 13 years of age. DL signers had been exposed to visual/written English prior to onset of ASL, although some reported not having acquired functional proficiency in it. None of the DE or DL participants knew any other sign language. Most were recruited at Gallaudet University in Washington, DC; the remainder were from New England, mainly Connecticut.

Hearing participants had normal hearing, were native English speakers, and did not speak any other language. HL participants,

Table 1

Characteristics of Each Participant Group

\begin{tabular}{|c|c|c|c|c|c|c|c|c|c|c|}
\hline \multirow[b]{2}{*}{ Group } & \multirow[b]{2}{*}{ Gender } & \multicolumn{3}{|c|}{ Age at Test (Years) } & \multicolumn{3}{|c|}{ AoA } & \multicolumn{3}{|c|}{$\begin{array}{l}\text { ASL Experience } \\
\text { (Years) }\end{array}$} \\
\hline & & $M$ & $S E$ & Range & $M$ & $S E$ & Range & $M$ & $S E$ & Range \\
\hline $\mathrm{DE}$ & $7 \mathrm{~F} / 3 \mathrm{M}$ & 31.1 & 3.4 & $18-48$ & 0 & 0 & $0-0$ & 31.1 & 3.4 & $18-48$ \\
\hline DL & $6 \mathrm{~F} / 4 \mathrm{M}$ & 29.7 & 2.4 & $19-44$ & 17.2 & 0.6 & $14-20$ & 12.5 & 2.3 & $2-24$ \\
\hline $\mathrm{HL}$ & $5 \mathrm{~F} / 5 \mathrm{M}$ & 27.4 & 2.7 & $21-45$ & 20.2 & 1.5 & $16-30$ & 7.2 & 2.3 & $3-25$ \\
\hline $\mathrm{HN}$ & $8 \mathrm{~F} / 2 \mathrm{M}$ & 30.3 & 3.1 & $21-44$ & $\mathrm{n} / \mathrm{a}$ & $\mathrm{n} / \mathrm{a}$ & $\mathrm{n} / \mathrm{a}$ & 0 & 0 & $0-0$ \\
\hline
\end{tabular}

Note-DE, deaf early signers; DL, deaf late signers; HL, hearing late signers; HN, hearing signnaive perceivers; AoA, onset age of acquisition of ASL. 


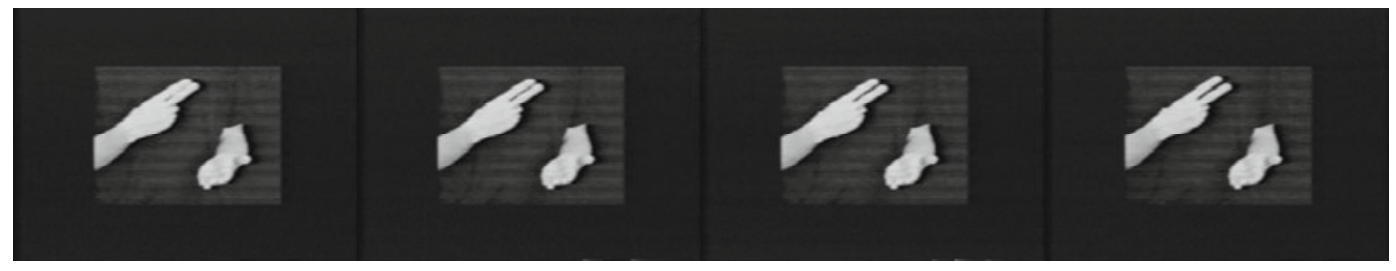

0

1

2

3

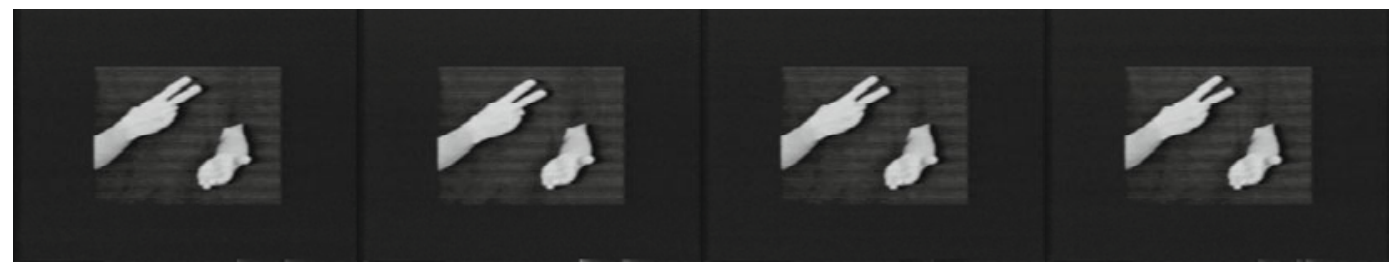

4

5

6

7

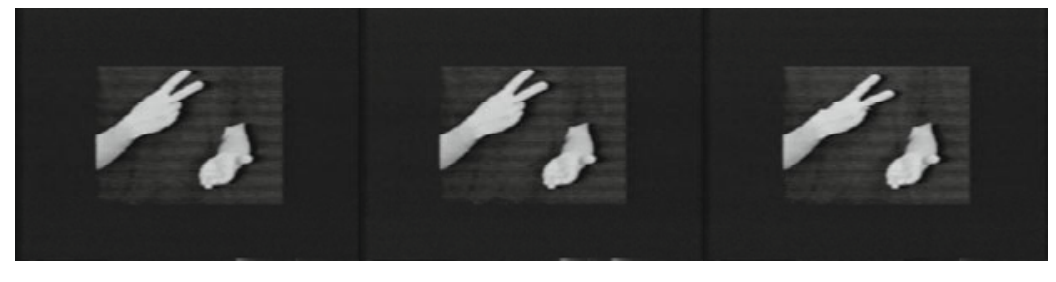

8

9

10

Figure 4. The continuum of equal-step differences in handshapes between $U$ and $V$.

like DL ones, started learning ASL after 13 years of age and were ASL-fluent by self-report. HN participants had no exposure to ASL or any other sign language. Hearing participants were recruited from Wesleyan University, the University of Connecticut, or surrounding towns in Connecticut. All participants had normal or corrected vision; none of them or their immediate family members had reading or language impairments. Most HN participants received experimental credit in introductory psychology; all other participants were paid for their participation.

\section{Stimuli}

An 11-item continuum of dynamic pseudosigns was developed around the minimal handshape contrast U-V (Figure 4). These handshapes were chosen for their visual and articulatory similarity. In both, the index and middle fingers are extended, but the spacing between them differs. In the $U$ handshape the fingers are in contact throughout their length ( $0 \%$ open; i.e., closed), but in the $\mathrm{V}$ handshape the fingers are spread $(100 \%$ open $) .^{2}$

$\mathrm{U}-\mathrm{V}$ minimal sign pairs exist in ASL, so this handshape distinction constitutes a true phonological contrast. A minimal pair that has a structure comparable to that of the target pseudosigns are particular variants of READ versus BUTTER (or LICK), both two-handed signs in which the tips of the extended index and middle fingers of the dominant hand are stroked down the open (flat) palm of the vertically oriented nondominant hand (B-handshape). The only difference between the two signs is handshape - specifically, whether the extended fingers of the dominant hand are spread apart (V-hand: READ) or not (U-hand: BUTTER/LICK).

The $\mathrm{U}$ and $\mathrm{V}$ handshapes were incorporated into phonotactically permissible, meaningless pseudosigns. ${ }^{3}$ We used the location, movement, and orientation of the ASL sign for STOP but replaced its dominant handshape (B-palm, facing signer) with $\mathrm{U}$ and $\mathrm{V}$ to make our endpoint pseudosigns (see Figures 5A and 5B). A skilled, hearing signer was videotaped as he executed those endpoints 20 times in a controlled manner, holding speed, hand positions, and orientation constant. He wore a black T-shirt to optimize the visibility of his hand and forearm. The video was digitized frame by frame as PICT files to a Macintosh computer, using QuickImage and a Sony SLV-676UC videorecorder. All tokens were carefully examined in Adobe Premiere to select a clearly articulated U-V pair that was best-matched in terms of number of frames ( $n=12$ each), hand position, orientation, movement pattern and speed, and other visual details.

The program Morph (Gryphon) was employed to create 9 distinct handshape images that differed in equal $10 \%$ increments of finger spreading between the $U$ ( $0 \%$ open: Item 0 on the continuum) and V (100\% open: Item 10 on the continuum) endpoints, for each corresponding pair of frames from the selected tokens. The resulting 108 morphed images (12 frame pairs $\times 9$ increments) were edited in Adobe Photoshop to delete any interfinger "webbing" created by the morphing process. The polished images were sequenced in correct temporal order for each of nine 12 -frame videos, creating a continuum of pseudosigns that varied in precise $10 \%$ increments of finger spreading between the $\mathrm{U}$ and $\mathrm{V}$ endpoints (for a diagram of the process, see Figure 6). These series were made into digital movie clips (each $\sim 0.4 \mathrm{sec}$ in duration) in Macromedia Director, which were then compiled into the trial sequences for the $\mathrm{AXB}$ discrimination and categorization tasks (for descriptions, see the Procedure section) and played from a Macintosh computer onto two separate videotapes. In both tasks, a dynamic visual masker appeared for $1 \mathrm{sec}$ after all three trial items to minimize the influence of iconic memory; that is, the masker, like the stimulus items, involved motion because its intended purpose was to mask any visual persistence of the preceding item, including its dynamic properties. The masker was "Primitive No Erase" (Macromedia Director), in which concentric white and gray flattened ellipses expand upward into circles, then collapse back 
A

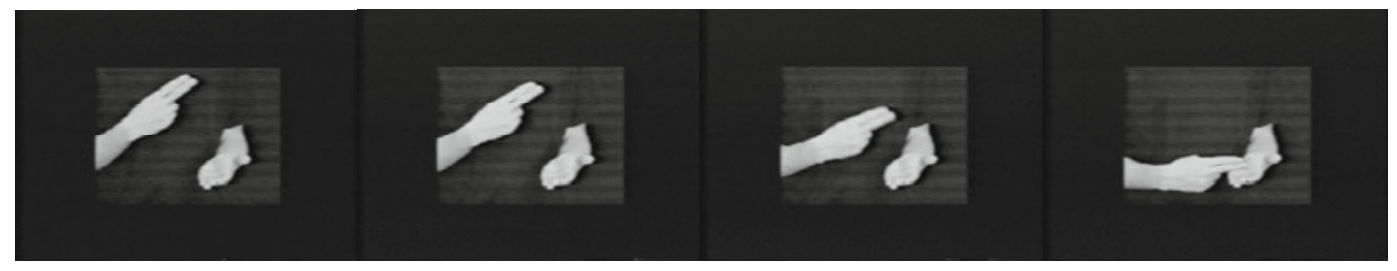

B

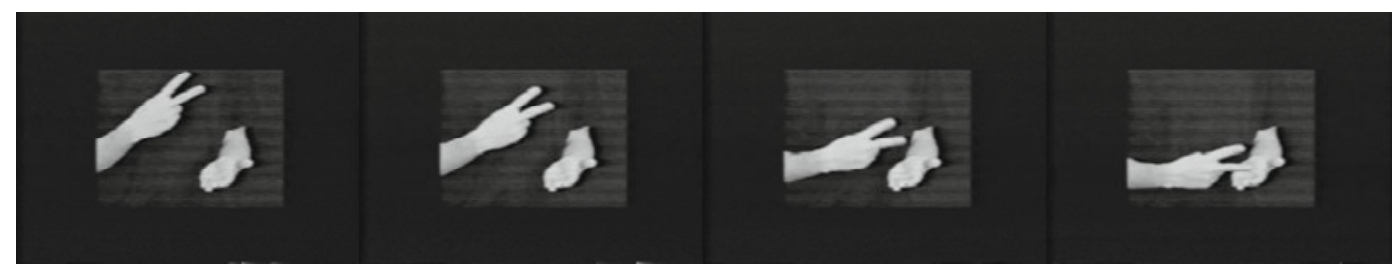

Figure 5. (A) Selected frames of final selected video for dynamic pseudosign STOP/U handshape. (B) Selected frames of final selected video for dynamic pseudosign STOP/V handshape.

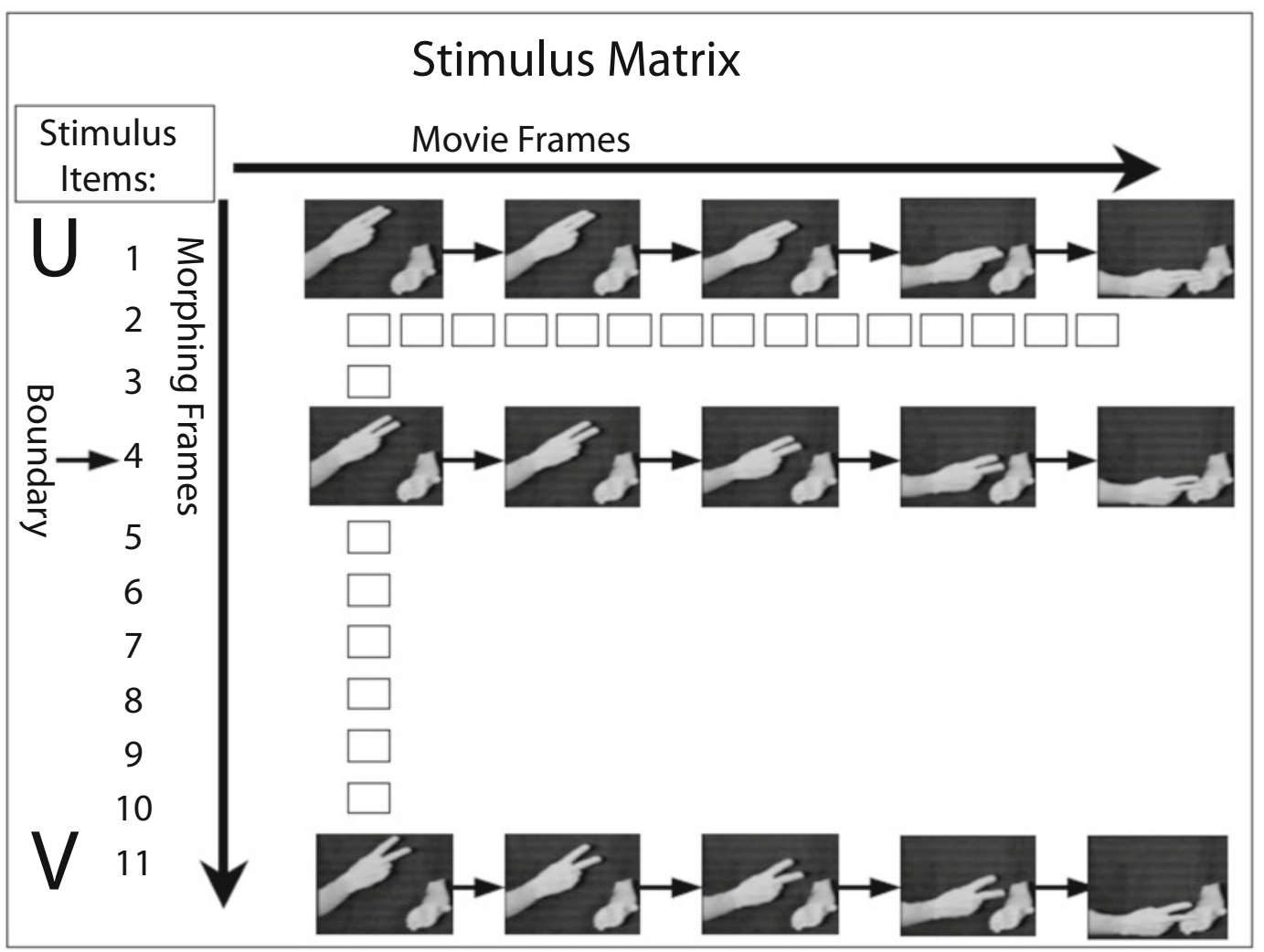

Figure 6. Matrix showing reconcatenation of frames for movie compilation of each dynamic pseudosign on the U-V 11-step continuum.

into flattened ellipses in a sequence that suggests an upward then downward motion against a black background. In both tasks, interstimulus intervals were $1 \mathrm{sec}$, intertrial intervals were $8 \mathrm{sec}$ of black screen, and interblock intervals were 12 sec of black screen.

\section{Procedure}

Participants signed a written-English informed consent form (which was signed in ASL to deaf participants) and completed a written background questionnaire on hearing status and history of language experience, before completing the two video tests, which were presented on a color video monitor via videotape. Each test was preceded by instructions on the videotape in ASL. Verbal instructions were provided for the hearing participants, and all participants were also given written English instructions for each task. After completing both tasks, in which the participants recorded their responses on answer sheets, all participants were given a written 
English debriefing sheet, as well as a verbal explanation in the appropriate language modality.

AXB discrimination. The discrimination task was conducted first to minimize any potential effects that categorizing the stimuli (the second task) may have imposed on discrimination performance. Trials were presented in a two-step AXB discrimination format. On each trial, A and B were continuum items separated by a $20 \%$ difference in finger spreading; there were nine pairings, from Items $0-2$ to Items $8-10$. The middle sign, $X$, was identical to either A or B, yielding four presentation triads for each two-step pairing $(\mathrm{AAB}, \mathrm{ABB}$, BAA, BBA; e.g., Item Triads $0-0-2,0-2-2,2-0-0$, and 2-2-0). Trials were presented in random order in eight blocks of 9 trials. Each trial type $(9$ pairs $\times 4$ triads $=36)$ was presented twice $(72$ trials). Participants judged whether $\mathrm{X}$ matched $\mathrm{A}$ or $\mathrm{B}$ after viewing the entire trial, including all visual maskers. Four extra trials at the start of the task served as practice; these were excluded from analyses.

The four triads per stimulus pair permit evaluation of primacy versus recency short-term memory effects in discrimination. Significantly better performance on $\mathrm{AAB}$ and $\mathrm{BBA}$ than on $\mathrm{ABB}$ and BAA triads reflects a primacy effect, interpreted as a linguistic bias in memory that arises from encoding language-specific information in the first stimulus. Better performance on $\mathrm{ABB}$ and BAA trials, on the other hand, reflects retention of the final stimulus in short-term perceptual memory (Crowder, 1982, 1993).

AXB categorization with goodness ratings. In this task, A and $B$ were always the $U$ (Item 0 ) versus the $V$ (Item 10) endpoint pseudosigns, respectively, whereas $\mathrm{X}$ was any one of the 11 items from the full continuum (e.g., 0-0-10, 0-2-10, or 0-9-10). Participants had to judge whether $\mathrm{X}$ was a member of Category A or Category $\mathrm{B}$. The trials were randomized and presented in 11 blocks of 10 trials each, with each continuum item presented as X 10 times ( $n=110$ trials). In addition, for each trial, the participants rated, on a scale of $1-4$, how well the middle item matched their chosen category $(1=$ poor; $4=$ excellent). Four extra trials at the start of the task again served as practice and were excluded from analyses. Although this task was completed after AXB discrimination, its results will be presented first to provide a context for evaluating discrimination.

\section{RESULTS}

\section{AXB Categorization and Goodness Ratings}

Because the classic definition of categorical perception is that discrimination is much better across a perceived category boundary than it is within categories, we report first on the analyses of the categorization and rating data (see Figure 7), even though those tasks were conducted after the discrimination test. To compare these data across groups, we conducted three overlapping pairwise sets of planned contrasts to systematically test the effects of signed and spoken language experience, and of hearing status, on perception of the target pseudosign contrast. (1) The DE versus DL comparisons evaluated the effect of


Figure 7. Mean percentage categorization as the 0 target (U handshape) and mean goodness rating for each continuum item by deaf (left: more experienced DE above) and hearing (right: more experienced HL above) groups. Solid black lines are the categorization functions; dashed lines are the rating functions. Error bars denote standard errors of the means. 
early (native) versus late (nonnative) acquisition of ASL among deaf participants - that is, with hearing status held constant. (2) The DL versus HL comparisons evaluated the effect of limited (deaf) versus normal access (hearing) to spoken English among late learners of ASL - that is, with nonnative, late-onset sign acquisition as the constant. (3) The HL versus HN comparisons evaluated the effect of substantial experience versus no experience with ASL among native English hearing participants - that is, with hearing and spoken native language as constants. Since this met the degrees of freedom constraint for planned contrasts ( $d f=n-1$, where $n=4$ groups), alpha level was set at .05 , without need for correction. We report results here for four measures of the AXB categorization task: $\mathrm{U}$ versus $\mathrm{V}$ categorization of the continuum items, ratings of the continuum items, the $50 \%$ intercept of the $\mathrm{U}-\mathrm{V}$ boundary, and the slope of the boundary around the intercept.

Two-way planned contrasts were conducted on the categorization functions for each designated pair of groups (between subjects) $\times 10$ stimulus items along the continuum (within subjects; the $\mathrm{V}$ endpoint [Stimulus Item 10] was omitted to maintain the $d f$ constraint), using percent identification of each continuum item as $\mathrm{U}(0 \%$ spread between index and middle fingers) as the dependent variable. Mean percent of $\mathrm{U}$ categorizations differed between the DE and DL groups $\left(M_{\mathrm{DL}}=34.55 \%\right.$; $\left.M_{\mathrm{DE}}=44.73 \%\right)[F(1,36)=7.689, p<.009]$, who diverged significantly for Stimulus Items $2\left(M_{\mathrm{DE}}=100 \%\right.$; $\left.M_{\mathrm{DL}}=76 \%\right)[F(1,36)=9.349, p<.004]$ and 3 of the continuum $\left(M_{\mathrm{DE}}=87 \% ; M_{\mathrm{DL}}=55 \%\right)[F(1,36)=8.393, p<$ $.006]$ and marginally for Stimulus Item $1\left(M_{\mathrm{DE}}=100 \%\right.$; $\left.M_{\mathrm{DL}}=90 \% \mathrm{U}\right)[F(1,36)=3.905, p<.056]$ and Stimulus Item $5\left(M_{\mathrm{DE}}=33 \% ; M_{\mathrm{DL}}=15 \%\right)[F(1,36)=3.369, p<$ $.075]$. The DL and HL groups also differed from one another in overall $\mathrm{U}$ categorizations $\left(M_{\mathrm{DL}}=34.55 \% ; M_{\mathrm{HL}}=\right.$ $45.09 \%)[F(1,36)=8.248, p<.007]$, significantly so on Stimulus Item $2\left(M_{\mathrm{DL}}=76 \% ; M_{\mathrm{HL}}=99 \%\right)[F(1,36)=$ $8.586, p<.006]$, Stimulus Item $3\left(M_{\mathrm{DL}}=55 \% ; M_{\mathrm{HL}}=\right.$ $97 \%)[F(1,36)=14.459, p<.001]$, and Stimulus Item 9 $\left(M_{\mathrm{DL}}=0 \% ; M_{\mathrm{HL}}=3 \%\right)[F(1,36)=7.714, p<.009]$, and marginally on Stimulus Item $1\left(M_{\mathrm{DL}}=90 \% ; M_{\mathrm{HL}}=\right.$ $100 \%)[F(1,36)=3.905, p<.056]$. In contrast, the two hearing groups HL (fluent nonnative ASL) and HN (no sign language experience) performed quite similarly, differing only on Stimulus Item $9\left(M_{\mathrm{HL}}=3 \% ; M_{\mathrm{HN}}=0 \%\right)$ $[F(1,36)=7.714, p<.009]$.

The analogous two-way planned contrasts on the goodness-of-fit ratings yielded compatible results. As expected, the overall picture is that the ambiguously categorized items near the category boundary were rated as mediocre to poor ( $\leq 2$ on the 4-point scale), whereas those near the endpoints received near-ceiling ratings $(>3.5$ : excellent). Where the categorization functions differed for DE-DL and DL-HL comparisons, ratings also differed among the groups for items near the boundaries. DE and DL ratings differed significantly on Stimulus Item 2 $\left(M_{\mathrm{DE}}=2.28 ; M_{\mathrm{DL}}=1.75\right)[F(1,36)=4.247, p<.047]$ and quite marginally on Stimulus Item $3\left(M_{\mathrm{DE}}=2.10\right.$;
$\left.M_{\mathrm{DL}}=1.67\right)[F(1,36)=2.947, p<.095]$, whereas DL and $\mathrm{HL}$ ratings differed significantly on Stimulus Item 2 $\left(M_{\mathrm{DL}}=1.75 ; M_{\mathrm{HL}}=2.45\right)[F(1,36)=7.353, p<.010]$ and marginally on Stimulus Item $1\left(M_{\mathrm{DL}}=2.72 ; M_{\mathrm{HL}}=\right.$ 3.18) $[F(1,36)=3.109, p<.086]$. By comparison, no effects approached significance for HL-HE.

To more closely probe the category boundary characteristics of the groups, PROBIT ogive curve-fitting analyses on the 11-step categorization functions were conducted to estimate each participant's U-V category boundary $(50 \%$ intercept) and the steepness of the categorization function around that boundary, or slope (see MacKain et al., 1981). The DE and DL groups differed significantly in boundary locations $\left(50 \%\right.$ intercept: $\left.M_{\mathrm{DE}}=3.72 ; M_{\mathrm{DL}}=2.29\right)$ $[F(1,36)=9.877, p<.003]$, and the DL and HL groups differed marginally $\left(M_{\mathrm{DL}}=2.29 ; M_{\mathrm{HL}}=4.68\right)[F(1,36)=$ $3.549, p<.068]$. Again, though, the $\mathrm{HL}$ and $\mathrm{HN}\left(M_{\mathrm{HN}}=\right.$ $3.44)$ groups failed to differ reliably.

The slope of the function around the U-V category boundary was less sensitive to group differences, showing a very weak but similar pattern as the other measures: greater differentiation between DE-DL than between DL-HL or HL-HN. DE and DL slopes differed marginally $\left(M_{\mathrm{DE}}=-1.10 ; M_{\mathrm{DL}}=-0.99\right)[F(1,36)=3.511, p<$ $.069]$, whereas neither the DL-HL group $\left(M_{\mathrm{HL}}=-1.31\right)$ nor the HL-HN group $\left(M_{\mathrm{HN}}=-1.13\right)$ comparisons showed even marginal differences.

\section{AXB Discrimination}

Percent correct discrimination for each stimulus pair was plotted for each group, and curve-fitting analyses were applied. Correlations between observed and fitted data indicated that polynomial functions provided the best fit for each group $\left(R^{2}\right.$ values for DE, DL, HL, and $\mathrm{HN}$ groups were quite high, at $.87, .95, .81$, and .93 , respectively). The best-fit polynomial function was linear for the DE and HN groups, both of whose members were native users of their respective languages, whereas the bestfitting function was curvilinear for the DL and HL groups, both late nonnative ASL users (see Figure 8).

Notably, no group showed the classic categorical (speech) perception pattern of peak discrimination across the category boundary and poor within-category performance on either side of the peak. An initial omnibus ANOVA revealed no significant contributions of AXB trial type (AAB, ABB, BBA, BAA), so this factor was collapsed for the planned pairwise group contrasts for DE-DL, DL-HL, and HL-HN. Because categorical perception predicts better discrimination at the categorization boundary than within categories, for these comparisons we reduced the data to three critical stimulus pairs: within-U $(0,2)$, within- $\mathrm{V}(8-10)$, and the "boundary" pair, defined for each participant as the stimulus pair that enclosed their PROBIT intercept (see Figure 9). To maintain the $d f$ constraint, we left out the within-V stimulus pair and included only the within- $U$ and boundary pairs in the group (2) $\times$ stimulus pair (2) planned contrasts. The dependent variable was percent correct discrimination. The DE and DL groups differed significantly on the bound- 



Figure 8. Mean percentage of correct discrimination across stimulus pairs, for all four groups. The solid black lines are the observed discrimination functions; dotted lines are the best-fit polynomial functions. Error bars denote standard errors of the means.

ary pair $\left(M_{\mathrm{DE}}=75 \% ; M_{\mathrm{DL}}=93.75 \%\right)[F(1,36)=4.655$, $p<.038]$. By comparison, the DL and HL groups differed only marginally, and on the within-U stimulus pair $\left(M_{\mathrm{DL}}=\right.$ $\left.97.50 \% ; M_{\mathrm{HL}}=85 \%\right)[F(1,36)=3.797, p<.059]$, not the

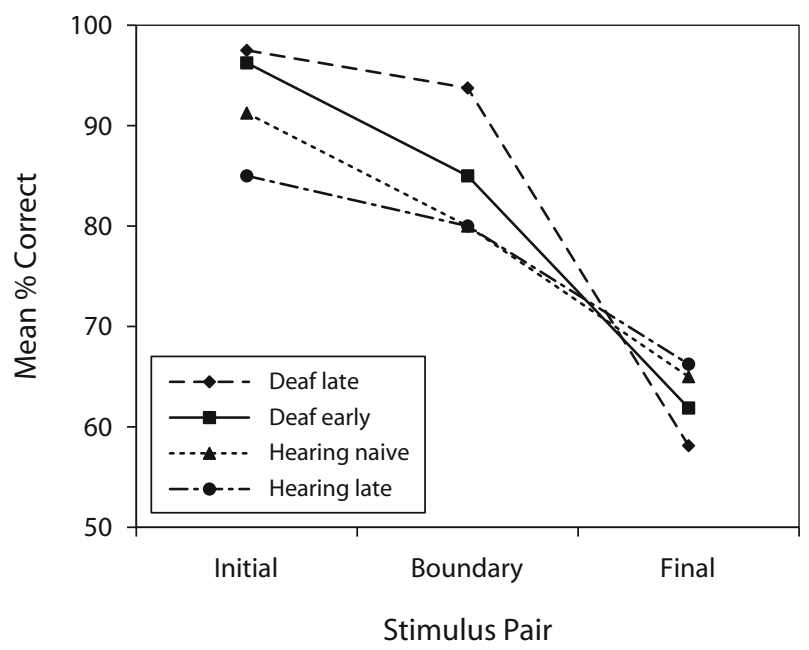

Figure 9. Mean percentage of correct discrimination for initial, boundary, and final pairs, by each group. boundary pair. HL and HN groups, conversely, showed no significant or even marginal differences. Thus, compatibly with the pattern of group differences found in the planned contrasts on the categorization/rating data, once again the clearest difference was found between the DE and DL groups, with a weaker difference between the DL and HL groups and no difference between the HL and HN groups.

However, the discrimination function for each group fails to follow the classic categorical perception pattern of highest performance across the perceived category boundary and poor within-category discrimination. Instead, the discrimination curves appear to reflect a psychophysical power function - that is, best discrimination of the within-U pair 0-2 (closed vs. slightly spread) and increasingly poorer discrimination of differences in relative finger-spreading toward the $\mathrm{V}$ end of the continuum - suggesting a progressive increase in just noticeable difference. Because the functions might be akin to the logarithmic changes in perceived pitch (mel scale) or loudness associated with linear increases along the physical dimensions of frequency and intensity (decibel [dB] scale), we applied a log transform to the data and reanalyzed, but this revealed no changes in the results from the untransformed data. 


\section{DISCUSSION}

The categorization functions for the $\mathrm{U}$ versus $\mathrm{V}$ phonological contrast, in the context of phonotactically permissible dynamic pseudosigns, were similar to those seen in speech research on categorical perception of minimal consonant contrasts; that is, overall, participants showed a regular, ogive-shaped curve with a relatively sharp U-V category boundary. Most relevant to the study's focus on language experience effects on perception of minimal sign contrasts is that the shape of the identification function, as well as the location and the steepness of the category boundary, varied significantly with ASL experience. The ratings of goodness of fit of the continuum items to the endpoint categories likewise indicated systematic effects of language experience quite consistent with the categorization findings.

Discrimination of two-step pairings of the stimuli along the continuum showed the same pattern of group differences as did the categorization data, reflecting analogous language experience effects. Note, however, that the shape of the discrimination functions differed markedly from those found in categorical perception studies with either consonants or vowels. None of the groups showed a peak in discrimination at the category boundary. Instead, all of their discrimination functions suggested a strong psychophysical component, in that the within-U pairs were discriminated quite well and performance decreased systematically to near-chance as the pairs approached the $\mathrm{V}$ end of the continuum. Nonetheless, the shape of the discrimination functions still showed three experience-related differences among the groups. First, the best-fit polynomial function was linear for the DE and $\mathrm{HN}$ groups but curvilinear for the HL and DL groups; that is, the shape diverged according to an aspect of language experience that, interestingly, superseded both hearing status and native language. Specifically, the two groups that were native users of their respective languages, one deaf and ASL native (DE), the other hearing and spoken English native (HN), showed a constant decline in sensitivity to a constant difference in degree of finger spreading, as the stimuli moved from the $\mathrm{U}$ end to the $\mathrm{V}$ end of the continuum. In contrast, the two late, nonnative signer groups, one hearing (HL) and the other deaf (DL), showed an exponential decrease in sensitivity as the stimulus pairs moved away from the $\mathrm{U}$ end and toward the $\mathrm{V}$ end. Second, when analyses were restricted to the extreme within- $U$ pair versus each participant's category boundary pair, discrimination was affected by hearing status. Within-U performance was indistinguishable between the two deaf groups and was also indistinguishable between the two hearing groups, but was significantly better for deaf than for hearing participants. Third, between-category (boundary pair) discrimination was, instead, affected by native versus (late) nonnative ASL acquisition (which was differentiated only in the deaf participants). Nonnative (late) deaf signers discriminated across the $\mathrm{U}-\mathrm{V}$ boundary better than did native deaf signers.

To summarize the findings across tasks, then: No significant differences emerged between the $\mathrm{HN}$ and non- native ASL-L2 HL groups on either task, regardless of measure. Thus, late ASL acquisition appears to have little to no impact on perception of phonetic detail in this ASL handshape contrast by hearing perceivers for whom spoken English is a well-established L1. However, late ASL acquisition clearly did affect categorization and discrimination for the deaf participants. DL signers were less likely than either DE signers or HL signers to categorize stimuli near the $\mathrm{U}$ endpoint as $\mathrm{U}$, or to rate them as good. Their category boundary was closer to the $\mathrm{U}$ end and shallower than those of the DE and HL groups. Conversely, their discrimination was more accurate than was that of deaf native signers near the boundary and was marginally more accurate than that of hearing nonnative signers for the within-U stimulus pair.

Why would DL ASL signers out-discriminate the DE and HL groups? One possibility is that, even for adult learners, increased experience with an L2 often leads to some improvement in categorizing and discriminating its phonetic contrasts (e.g., MacKain et al., 1981; Miyawaki et al., 1975; Werker \& Lalonde, 1988). Given that ASL is the primary mode of communication for DL signers and that spoken English is primary for HL signers, it follows that DL signers should discriminate ASL contrasts better than HL signers, as they indeed do. However, this account should also predict that DE signers, who have acquired ASL from infancy and have far more ASL experience than do DL signers, would outperform them on discrimination of minimal sign contrasts. Yet the opposite is true: DL signers out-discriminate DE signers, especially at the region of the category boundary, and show correspondingly finer differentiation in their categorization and ratings of stimulus variations at the $U$ end of the continuum.

Thus, we must consider alternative explanations. One important factor to consider is that most DL signers generally do not achieve fluent L1 acquisition of lipread/written English comparable to that of hearing native-English individuals, even with extensive oral language training and speech therapy. Thus, they may actually be best considered as late functional L1-sign learners, a situation that poses unique difficulties in language learning and perception that lead them to attend more to the fine-grained phonetic properties of signs, including phonologically irrelevant details, than do DE signers or, indeed, either of the native-English hearing groups. This picture is consistent with prior reports that DL signers make significantly more phonological errors in sign recall than do DE signers, who make more semantic errors (Mayberry \& Fischer, 1989). In fact, there is a tendency for phonological errors to increase with the AoA of ASL (Mayberry \& Eichen, 1991). Those findings have been interpreted to indicate that native signers automatically encode a sign's phonological form, thus gaining rapid access to the lexical item and its semantics, rather than focusing on its surface form. DL signers encode sign forms less efficiently and less automatically, such that surface phonetic variations present a processing bottleneck that results in confusion of phonetically similar phonological features. This is compatible with the fact that they discriminate fine phonetic distinctions better than DE signers do (Mayberry, 2007). 
The planned comparisons we conducted provided a more focused and appropriate analytic approach than a standard ANOVA could have, given the nonequivalence across the deaf and hearing groups along the possible language experience dimensions one could have considered using for a factorial analysis. However, there are a number of caveats, even with respect to the planned contrasts. First, as the preceding paragraph indicates, hearing versus deaf late nonnative signers will nearly always differ both in L2-ASL experience, on the one hand, and in L1-English fluency, on the other. HL signers use ASL notably less often than do DL signers and, conversely, rely much more on spoken English for their daily communication. Second, DL and HL signers also, of course, differ in hearing status. This makes it difficult to determine the extent to which group differences in perception of sign contrasts result from differential experience with sign language, as opposed to differential access to auditory information about spoken language. Third, and relatedly, both hearing groups' perception of sign contrasts may be strongly affected by having spoken English as a native language, but for severely or profoundly deaf participants, this would not be the case, whether they are DE signers or DL signers. Finally, DL signers as a group are inherently more heterogeneous in their language learning and usage than are the other groups. Only a few are likely to have established a strong prior foundation in spoken and/ or written English and to remain dominant in English, like HL signers. The majority are likely to shift to dominant use of ASL. Given this heterogeneity, it is not surprising that the DL group were more variable in their categorization and rating performance than the other groups were (see error bars in Figure 7).

Two additional groups could help to sort out the contributions of hearing status, speech experience, and sign experience: hearing native signers and deaf sign-naive participants. Hearing native signers are the offspring of native-signing deaf adults and learn sign language from birth (also known as "CODAs"-i.e., children of deaf adults). Despite their normal hearing, sign is their native language, and they often show some delay in acquisition of spoken language. Meanwhile, deaf sign-naive participants typically have hearing/speaking parents and have been placed in an educational context that emphasizes the acquisition of spoken English without the use of any sign language (the "oral method"). Although many do meet deaf people later in life from whom they learn sign, some do not and continue to use English (lipread and/or written) as their only language.

The present findings must also be considered in light of previous reports on categorical perception of sign contrasts. A core question is why we, as well as Newport (1982) and Morford et al. (2008), failed to find the classic pattern of categorical perception of minimal handshape contrasts in native signers, whereas the other two prior studies reported finding it for some handshape contrasts (Baker et al., 2005; Emmorey et al., 2003). Among the potential reasons for the discrepant findings, as discussed earlier, are stimulus and methodology differences. To focus more closely on the stimulus issue, one possibility is that the inconsistent results are related to variations in psychophysical effects for the different handshape contrasts. We found greater sensitivity to $20 \%$ differences in finger spreading at the $U$ end (touching) of the continuum than at the $V$ end (spread) for all groups, suggesting a sizable psychophysical contribution to perception of this sign contrast. Lateral contact between the fingers presents a visual and articulatory boundary condition from which any small deviation in spreading at the metacarpophalangeal joints (knuckles at the interface of fingers and palm) may be easier to detect than equal amounts of difference among more open (i.e., already spread) configurations.

Maybe this sort of asymmetrical psychophysical boundary effect could account for even the native signers' failure to show categorical discrimination of $\mathrm{U}-\mathrm{V}$, which differs solely in $+/-$ spreading between fully extended (-flexed) fingers. If so, psychophysical asymmetries may also apply to other single-feature handshape distinctions. For example, the $\mathrm{X}$ versus 1 (Figure 1) contrast (Newport, 1982) also failed to yield categorical discrimination in signers, even though the target stimuli in their first experiment, like our stimuli, were dynamic and phonotactically permissible (indeed, Newport's [1982] stimuli were real signs). That handshape contrast involves a different feature than $\mathrm{U}-\mathrm{V}$ - specifically, + /-flexed: The selected finger (index) has the interphalangeal joints (knuckles on the fingers) either fully flexed (closed) or fully extended (straight). However, it would be reasonable to hypothesize that psychophysical sensitivity to small deviations from straight extension (1) is greater than that for equalmagnitude deviations from its opposite, full flexion (X).

That reasoning might seem to apply also to A-bar versus B-bar, which contrast on whether all four laterally touching fingers ( - spread) are flexed or extended, and the 5 versus 3 contrast, which differ in number of fingers extended $(5=$ thumb and all fingers; $3=$ only thumb, index, and middle finger; see Figure 2). But, in fact, both A-bar/B-bar and 5/3 actually involve two articulatory feature distinctions and, thus, differ from the single-feature $\mathrm{U}-\mathrm{V}$ and $1 / \mathrm{X}$ cases. For A-bar versus B-bar, the feature $+/-$ flexed differs for all four fingers at two sets of quasi-independently controllable joints (interphalangeal vs. metacarpophalangeal), whereas 5 versus 3 differ on those same two dimensions, but only with respect to the ring finger and pinkie. In light of these articulatory considerations, it is noteworthy that native signers have been reported to categorically perceive static images of 5 versus 3 (Emmorey et al., 2003), as well as static (Emmorey et al., 2003), dynamically rotating A- versus B-bar handshapes (Baker et al., 2005), and dynamic synthetic stimuli that used A- versus B-bar handshapes (Morford et al., 2008). ${ }^{4}$ Those observations raise the possibility that categoricity in perception of sign handshapes might be related to whether the contrast is truly minimal (single feature) or, instead, involves distinctions in more than one articulatory feature.

Let us consider that possibility for the contrasts 5 versus $\mathrm{S}, 5$ versus flat-O (Baker et al., 2005), B-bar versus claw, flat-O versus 8 (Morford et al., 2008) and (noncontrastive allophones) open-N versus closed-N (Emmorey et al., 2003). The first four involve more than one articulatory feature difference (see Figure 2). The 5/S pair dif- 
fer on $+/-$ flexion in both the interphalangeal and the metacarpophalangeal joints of all fingers and thumb. In 5/flat-O, both sets of joints are extended for the selected fingers in 5 (all fingers and thumb), whereas for flat-O they are flexed at only the metacarpophalangeal joints (i.e., the interphalangeals remain extended but the fingers themselves remain straight). In addition, for flat-O but not for 5 , the fingertips are in contact with the thumb. The B-bar/claw pair differ on whether the all the fingers are - spread (B-bar) or + spread (claw); they also differ on whether the fingers are fully extended (-flexion: B-bar) or flexed on the interphalangeal joints of all fingers and thumbs (claw). In the flat-O/8 pair, all fingers are flexed at the metacarpophalangeal joints and contact the thumb in flat-O. The difference with 8 is twofold: The selected fingers are different (only the middle finger is flexed at the metacarpophalangeal joint and touches the thumb), and the nonselected fingers (index, ring, and pinkie) are extended at the metacarpophalangeal joints. In contrast, for open-/ closed-N, the selected fingers (thumb, index, and middle fingers) are extended at the interphalangeals but flexed at the metacarophalangeals; these two (noncontrastive, or allophonic) handshapes differ only in whether the fingertips contact the thumb.

Might it be the case that multiple articulatory differences between handshapes can override the simple psychophysical effects of a singe-feature difference, allowing categorical perception to emerge? The answer is not entirely consistent: 5 versus flat-O was categorically perceived by signers (Baker et al., 2005), whereas 5 versus $S$ (Baker et al., 2005) and open- versus closed-N were not (Emmorey et al., 2003). Still, of the six tested handshape distinctions that differed on more than a single articulatory feature, five were reported to be categorically perceived (A-/B-bar (see note 4), 5/3, 5/flat-O, B-bar/claw, flat-O/8); only one was not $(5 / \mathrm{S})$. None of the singlefeature contrasts was categorically perceived $(\mathrm{U}-\mathrm{V}, 1 / \mathrm{X}$, open-/closed-N). We acknowledge that the current understanding of what constitutes a feature in sign language is rudimentary and tentative at best. At the same time, an important contribution of this report is that it weds hypotheses about phonetic structure in signs to careful perceptual experimentation.

The strong psychophysical aspect of $\mathrm{U}-\mathrm{V}$ discrimination, and its discrepancy from the expected pattern of maximum performance across the categorization boundary, might be understood in relation to other categorical perception findings in the visual domain. In color categorical perception, for example, the peak in the discrimination function does not stand out as much from the rest of the function, as has been found in categorical perception of consonant contrasts (Diehl \& Kluender, 1987), and instead better resembles categorical perception of vowels. As for prior reports on categorical perception of sign contrasts, Emmorey et al. (2003, p. 33) noted that their discrimination functions resemble the attenuated boundary peaks and good within-category performance found with other types of human visual stimuli, such as famous faces (Beale \& Keil, 1995) and emotional facial expressions (de Gelder, Teunisse, \& Benson, 1997; Etcoff
\& Magee, 1992). Emmorey et al. found excellent overall discrimination, rather than weak categorical discrimination, for certain other sign contrasts, which were unaffected by sign language experience. Moreover, although certain linguistically contrastive facial expressions used in ASL and British Sign Language are reportedly perceived categorically (Campbell, Woll, Benson, \& Wallace, 1999; McCullough \& Emmorey, 2009), this was also unaffected by sign experience; that is, it appeared in both deaf native signers and hearing nonsigners.

It may be that the differences between our findings and others in the visual domain, both for sign language and for nonlinguistic properties such as color categories, result from the fact that only our stimuli have combined all of the following properties employed in studies on categorical perception of speech: rapid, dynamic, human gestures, in phonotactically permissible nonsense items, which use a single articulatory difference to convey a linguistic contrast that can distinguish lexical items. These parallels also allow the most straightforward comparison with categorical perception findings on speech. In that light, we can conclude that for a minimal handshape contrast involving just a single articulatory distinction, categorization (and goodness ratings) parallels both the categoricity and the effects of language experience that have been found in categorical perception of minimal speech contrasts. And whereas discrimination of this contrast failed to parallel the discrimination functions found for minimal consonant, or even vowel, contrasts, it nonetheless showed a comparable pattern of experience-related group differences.

The categorization and discrimination functions are mutually consistent, if viewed from outside a strict categorical perception framework. All groups discriminated stimuli better at the $U$ end of the continuum and categorized fewer stimuli in the $\mathrm{U}$ category. Likewise, all groups discriminated stimuli at the $\mathrm{V}$ end of the continuum poorly and categorized those stimuli less variably. Furthermore, the group that was most sensitive to phonetic variability around the U endpoint (DL: deaf nonnative signers) was least consistent in categorizing those same stimuli.

Additional research is still needed to clarify the perceptual contributions of phonetic distinctions for all of the phonological parameters of signs. Categorical perception studies are only part of the toolbox available for probing the impact of experience on recognition of signs and minimal contrasts in sign languages. The categorical studies have focused mainly on handshape contrasts, although they have examined a more restricted number and type of location contrasts. The few location contrasts investigated in categorical perception studies have shown neither the categorical patterns seen in speech studies nor any effects of sign language experience (Emmorey et al., 2003; Morford et al., 2008; Newport, 1982). Movement contrasts, however, have not been examined yet via categorical perception techniques. Meanwhile, there is evidence from other types of tasks that signers perceive location and movement contrasts differently than handshape contrasts (Emmorey \& Corina, 1990; Poizner \& Lane, 1978; Stungis, 1981). Primed lexical decisions by signers are facilitated when the primes share location or movement 
with the target, but not when they share handshape (Dye \& Shih, 2006). Also, signers judge signs sharing location or movement to be more similar than those that share handshape (Corina \& Hildebrandt, 2002). However, all we know thus far about the perceptual effects of sign language experience on these two parameters is that, whereas deaf signers find linguistically significant movement features more salient than do hearing nonsigners (Poizner, 1983), location contrasts show no effect of experience, not being categorically perceived by either group.

In conclusion, the full classic categorical perception pattern does not occur for minimal handshape contrasts in sign language, even with stimuli and experimental conditions analogous to those used in speech research. Nonetheless, performance on the categorization, goodness rating, and discrimination tasks revealed a consistent pattern of experiential influences in handshape perception. Specifically, native versus late nonnative acquisition of sign language by deaf participants significantly affected perception of stimulus variations at the $U$ end of the continuum on all three tasks. However, for hearing participants with prior native fluency in spoken English, achieving late nonnative fluency in ASL did not reliably influence perception of the target handshape contrast on any of the three tasks. Several key questions remain unanswered about perception of the three primary phonological dimensions of signs, however, particularly with regard to the effects of early signed versus spoken language experience. Further research is needed on the perception of location and movement contrasts, using not only categorical perception techniques but others as well. We hope that the approach and findings of the present study will provide a useful foundation for further research on these issues.

\section{AUTHOR NOTE}

This research was supported by U.S. National Institutes of Health Grants HD-01994 (Project 3 leader: C.T.B.), HD-01994 postdoctoral supplement (G.M.), and DC-00403 (C.T.B.), and a University of Western Sydney (Australia) International Research Initiative Scheme (IRIS) grant (C.T.B.). Correspondence concerning this article should be addressed to C. T. Best, Haskins Laboratories, 300 George St., New Haven, CT 06511 (e-mail: c.best@uws.edu.au).

\section{REFERENCES}

Abramson, A. S., \& Lisker, L. (1970). Discriminability along the voicing continuum: Cross-language tests. In B. Hála, M. Romportl, \& P. Janota (Eds.), Proceedings of the 6th International Congress of Phonetic Sciences, Prague 1967 (pp. 569-573). Prague: Academia.

Bailey, P. J., \& Summerfield, A. Q. (1977). On the identification of sine-wave analogues of certain speech sounds (Rep. SR-51/52, pp. 1-25). New Haven, CT: Haskins Laboratories.

Baker, S. A., Idsardi, W. J., Golinkoff, R. M., \& Petitto, L.-A. (2005). The perception of handshapes in American Sign Language. Memory \& Cognition, 33, 887-904.

Battison, R. (1978). Lexical borrowing in American Sign Language. Silver Spring, MD: Linstok.

Beale, J. M., \& Keil, F. C. (1995). Categorical effects in the perception of faces. Cognition, 57, 217-239. doi:10.1016/0010 $-0277(95) 00669-X$

Best, C. T., McRoberts, G. W., \& Goodell, E. (2001). Discrimination of non-native consonant contrasts varying in perceptual assimilation to the listener's native phonological system. Journal of the Acoustical Society of America, 109, 775-794. doi:10.1121/1.1332378

Best, C. T., Morrongiello, B., \& Robson, R. (1981). Perceptual equivalence of acoustic cues in speech and nonspeech perception. Perception \& Psychophysics, 29, 191-211.

Best, C. T., \& Strange, W. (1992). Effects of phonological and phonetic factors on cross-language perception of approximants. Journal of Phonetics, 20, 305-330.

Best, C. T., Studdert-Kennedy, M., Manuel, S., \& Rubin-Spitz, J. (1989). Discovering phonetic coherence in acoustic patterns. Perception \& Psychophysics, 45, 237-250.

Bosworth, R., \& DobKINs, K. (2002). The effects of spatial attention on motion processing in deaf signers, hearing signers, and hearing nonsigners. Brain \& Cognition, 49, 152-169. doi:10.1006/brcg.2001.1497

BRENTARI, D. (1998). A prosodic model of sign language phonology. Cambridge, MA: MIT Press.

Campbell, R., Woll, B., Benson, P., \& Wallace, S. (1999). Categorical perception of face actions: Their role in sign language and in communicative facial displays. Quarterly Journal of Experimental Psychology, 52A, 67-95. doi:10.1080/027249899391232

Chang, Y.-C., Hallé, P., Best, C. T., \& Abramson, A. S. (2008, April). Do non-native language listeners perceive Mandarin tone continua categorically? Paper presented at the 8th Phonetic Conference of China and the International Symposium on Phonetic Frontiers, Beijing.

Corina, D., \& Hildebrandt, U. (2002). Psycholinguistic investigations of phonological structure in ASL. In R. P. Meier, K. Cormier, \& D. Quinto-Pozos (Eds.), Modality and structure in signed and spoken languages (pp. 88-111). Cambridge: Cambridge University Press.

Crowder, R. G. (1982). The demise of short-term memory. Acta Psychologica, 50, 291-323. doi:10.1016/0001-6918(82)90044-0

Crowder, R. G. (1993). Short-term memory: Where do we stand? Memory \& Cognition, 21, 142-145.

de Gelder, B., Teunisse, J.-P., \& Benson, P. J. (1997). Categorical perception of facial expressions: Categories and their internal structure. Cognition \& Emotion, 11, 1-23. doi:10.1080/026999397380005

DieHL, R. L., \& Kluender, K. R. (1987). On the categorization of speech sounds. In S. Harnad (Ed.), Categorical perception: The groundwork of cognition (pp. 121-144). Cambridge: Cambridge University Press. doi:10.1121/1.398286

Dye, M. W., \& Bavelier, D. (in press). Attention following early auditory deprivation: Temporal versus spatial selection. Restorative Neurology \& Neuroscience.

Dye, M. W., \& Shiн, S. (2006). Phonological priming in British Sign Language. In L. M. Goldstein, D. H. Whalen, \& C. T. Best (Eds.), Papers in Laboratory Phonology 8: Varieties of phonological competence (pp. 241-263). Berlin: Mouton de Gruyter. doi:10.1515/ 9783110197211.1.241

EMmorey, K., \& Corina, D. (1990). Lexical recognition in sign language: Effects of phonetic structure and morphology. Perceptual \& Motor Skills, 71, 1227-1252. doi:10.2466/PMS.71.8.1227-1252

Emmorey, K., McCullough, S., \& Brentari, D. (2003). Categorical perception in American Sign Language. Language \& Cognitive Processes, 18, 21-45. doi:10.1080/01690960143000416

Etcoff, N. L., \& Magee, J. J. (1992). Categorical perception of facial expressions. Cognition, 44, 227-240. doi:10.1016/0010-0277 (92)90002-Y

Ferrero, F. E., Pelamatti, G. M., \& Vagges, K. (1982). Continuous and categorical perception of a fricative-affricate continuum. Journal of Phonetics, 10, 231-244.

Francis, A., Ciocca, V., \& KeI, C. N. B. (2003). On the (non)categorical perception of lexical tones. Perception \& Psychophysics, 65, 10291044.

Fry, D. B., Abramson, A. S., Eimas, P. D., \& Liberman, A. M. (1962). The identification and discrimination of synthetic vowels. Language \& Speech, 5, 171-189.

FuJISAKI, H., \& KaWASHIMa, T. (1969). On the modes and mechanisms of speech perception (Annual Report of the Engineering Research Institute No. 28). Tokyo: University of Tokyo Faculty of Engineering. doi:10.1121/1.1974614

GaNONG, W. F. (1980). Phonetic categorization in auditory word perception. Journal of Experimental Psychology: Human Perception \& Performance, 6, 110-125. doi:10.1037/0096-1523.6.1.110

Hallé, P. A., Best, C. T., \& LevitT, A. (1999). Phonetic vs. phonological influences on French listeners' perception of American English approximants. Journal of Phonetics, 27, 281-306. doi:10.1006/jpho .1999 .0097 
Hallé, P. A., Chang, Y.-C., \& Best, C. T. (2004). Identification and discrimination of Mandarin Chinese tones by Mandarin Chinese vs. French speakers. Journal of Phonetics, 32, 395-421. doi:10.1016/S0095 $-4470(03) 00016-0$

HaRnAD, S. (ED.) (1987). Categorical perception: The groundwork of cognition. Cambridge: Cambridge University Press. doi:10.1121/ 1.398286

Hodgson, P., \& MiLler, J. L. (1996). Internal structure of phonetic categories: Evidence for within-category trading relations. Journal of the Acoustical Society of America, 100, 565-576. doi:10.1121/1.415867

IVERSON, P., \& KUHL, P. K. (1996). Influences of phonetic identification and category goodness of American listeners' perception of $/ \mathrm{r} /$ and /1/. Journal of the Acoustical Society of America, 99, 1130-1140. doi:10.1121/1.415234

JoAnisse, M., Zevin, J., \& McCANDliss, B. (2007). Brain mechanisms implicated in the preattentive categorization of speech sounds revealed using fMRI and a short-interval habituation trial paradigm. Cerebral Cortex, 17, 2084-2093. doi:10.1093/cercor/bhl124

Keating, P. A., Mikoś, M. J., \& Ganong, W. F. (1981). A crosslanguage study of range of voice onset time in the perception of initial stop voicing. Journal of the Acoustical Society of America, 70, 12611271. doi:10.1121/1.387139

Kuhl, P. K., Williams, K. A., Lacerda, F., Stevens, K. N., \& LindBLOM, B. (1992). Linguistic experience alters phonetic perception in infants by 6 months of age. Science, 255, 606-608. doi:10.1126/ science. 1736364

Liberman, A. M., Cooper, F. S., Shankweiler, D. P., \& StuddertKennedy, M. (1967). Perception of the speech code. Psychological Review, 74, 431-461. doi:10.1037/h0020279

Liberman, A. M., Harris, K. S., Hoffman, H. S., \& Griffith, B. C. (1957). The discrimination of speech sounds within and across phoneme boundaries. Journal of Experimental Psychology, 54, 358-368. doi: $10.1037 / \mathrm{h} 0044417$

LidDELl, S., \& JoHnSon, R. (1989). American Sign Language: The phonological base. Sign Language Studies, 64, 195-277.

MacKain, K. S., Best, C. T., \& Strange, W. (1981). Categorical perception of English /r/ and /1/ by Japanese bilinguals. Applied Psycholinguistics, 2, 369-390. doi:10.1017/S0142716400009796

MaYberRY, R. I. (1993). First-language acquisition after childhood differs from second-language acquisition: The case of American Sign Language. Journal of Speech \& Hearing Research, 36, 1258-1270.

MAYBERRY, R. I. (2007). When timing is everything: Age of firstlanguage acquisition effects on second-language learning. Applied Psycholinguistics, 28, 537-549.

Mayberry, R. I., \& Eichen, E. B. (1991). The long-lasting advantage of learning sign language in childhood: Another look at the critical period for language acquisition. Journal of Memory \& Language, 30, 486-512.

MaYberRy, R. I., \& Fischer, S. D. (1989). Looking through phonological shape to lexical meaning: The bottleneck of non-native sign language processing. Memory \& Cognition, 17, 740-754

McCullough, S., \& Emmorey, K. (2009). Categorical perception of affective and linguistic facial expressions. Cognition, 110, 208-221.

McQueen, J. M. (1996). Phonetic categorization. Language \& Cognitive Processes, 11, 655-664. doi:10.1080/016909696387060

MilleR, J. L. (1994). On the internal structure of phonetic categories: A progress report. Cognition, 50, 271-285. doi:10.1016/0010 $-0277(94) 90031-0$

Miyawaki, K., Strange, W., Verbrugge, R., Liberman, A. M., JenKINS, J. J., \& FuJIMURA, O. (1975). An effect of linguistic experience: The discrimination of $[\mathrm{r}]$ and [1] by native speakers of Japanese and English. Perception \& Psychophysics, 18, 331-340.

Morford, J., Grieve-Smith, A., MacFarlane, J., Staley, J., \& WATERS, G. (2008). Effects of language experience on the perception of American Sign Language. Cognition, 109, 41-53. doi:10.1016/ j.cognition.2008.07.016

NEWPORT, E. (1982). Task specificity in language learning? Evidence from speech perception and American Sign Language. In E. Wanner \& L. Gleitman (Eds.), Language acquisition: The state of the art (pp. 450-486). Cambridge: Cambridge University Press.

Pisoni, D. B. (1973). Auditory and phonetic memory codes in the discrimination of consonants and vowels. Perception \& Psychophysics, 13, 253-260.
Poizner, H. (1983). Perception of movement in American Sign Language: Effects of linguistic structure and linguistic experience. Perception \& Psychophysics, 33, 215-231.

Poizner, H., \& LANe, H. (1978). Discrimination of location in American Sign Language. In P. Siple (Ed.), Understanding language through sign language research (pp. 271-288). New York: Academic Press.

Repr, B. H., Healy, A. F., \& Crowder, R. G. (1979). Categories and context in the perception of isolated steady-state vowels. Journal of Experimental Psychology: Human Perception \& Performance, 5, 129145. doi:10.1037/0096-1523.5.1.129

Rosen, S., \& Howell, P. (1987). Auditory, articulatory, and learning explanations of categorical perception in speech. In S. Harnad (Ed.), Categorical perception: The groundwork of cognition (pp. 113-160). Cambridge: Cambridge University Press. doi:10.1121/1.398286

SANDLER, W. (1989). Phonological representation of the sign: Linearity and nonlinearity in American Sign Language. Dordrecht, The Netherlands: Foris.

SANDler, W., \& Lillo-Martin, D. (2006). Sign language and linguistic universals. Cambridge: Cambridge University Press.

Simon, C., \& Fourcin, A. J. (1978). Cross-language study of speechpattern learning. Journal of the Acoustical Society of America, 63, 925-935. doi:10.1121/1.381772

Simos, P. G., Diehl, R. L., Breier, J. I., Molis, M. R., Zouridakis, G., \& PAPANicolaou, A. C. (1998). MEG correlates of categorical perception of a voice onset time continuum in humans. Cognitive Brain Research, 7, 215-219. doi:10.1016/S0926-6410(98)00037-8

Stevens, K. N., Liberman, A. M., Öhman, S. E. G., \& StuddertKenNEDY, M. (1969). Crosslanguage study of vowel perception. Language \& Speech, 12, 1-23.

Stokoe, W., Casterline, D., \& Cronberg, C. (1965). A dictionary of American Sign Language on linguistic principles. Silver Spring, MD: Linstok.

StUngis, J. (1981). Identification and discrimination of handshape in American Sign Language. Perception \& Psychophysics, 29, 261-276.

WerkeR, J. F., \& LALONDE, C. E. (1988). Cross-language speech perception: Initial capabilities and developmental change. Developmental Psychology, 24, 672-683. doi:10.1037/0012-1649.24.5.672

WiLliams, L. (1977). The perception of stop consonant voicing by SpanishEnglish bilinguals. Perception \& Psychophysics, 21, 289-297.

\section{NOTES}

1. A hearing native-ASL group would have permitted evaluation of hearing status effects at the other extreme of ASL-onset age. Indeed, there is evidence that hearing status affects perception in the visual domain. For example, auditory deprivation has affected specific aspects of visual attention (Bosworth \& Dobkins, 2002; Dye \& Bavelier, in press). Unfortunately, we were unable to locate sufficient numbers of this population. In any case, the four groups included in our study nonetheless allow systematic comparison of the key experiential factors.

2. It must be noted that $V$ handshape in ASL could potentially be specified as any nonzero degree of opening between the fingers - in other words, as any spreading at all. No prior research has examined this issue.

3. Like many other handshapes, $\mathrm{U}$ and $\mathrm{V}$ can also be used as fingerspelled letters - in which case, they must have a specific orientation, movement, and location that do not occur in signs or in the stimuli here. Thus, there is a clear distinction between handshapes as fingerspelled letters versus in signs. We note, in addition, that the $\mathrm{V}$ handshape itself signifies Two. As with fingerspelled handshapes, it has a specific orientation, movement, and location that differ from its use in other signs and in our stimuli, regardless of whether it is produced in isolation or is a quantifier in signs like TWO-YEAR-OLD, TWO-DOLLAR. Thus, our target items are unequivocally pseudosigns: Neither is a fingerspelled letter, a lexical item, or a bound morpheme, but each is phonotactically permissible.

4. Morford et al. (2008) indicated that their findings for the A-bar B-bar contrast are consistent with those of Emmorey et al. (2003) and Baker et al. (2005). However, they did not interpret those findings as evidence for categorical perception, given their own findings on deaf versus hearing nonnative (late) signers.

(Manuscript received September 8, 2008; revision accepted for publication October 15, 2009.) 